\title{
Hepatoprotective effect of Prunus armeniaca L. (Apricot) leaf extracts on Paracetamol induced liver damage in Wistar rats
}

\author{
Varsha Raj", Arun Kumar Mishra, Amrita Mishra, Najam Ali Khan \\ Department of Pharmacy, Pharmacology Research Lab, IFTM University, Moradabad, 244102, INDIA.
}

\begin{abstract}
Objective: To evaluate the hepatoprotective effect of Prunus armeniaca L. (Apricot) leaf on paracetamol induced liver toxicity in rats. Method: Phytochemical investigation was performed to find active constituents of the plant extracts by the different phytochemical tests. After induction of liver toxicity, the biochemical parameters such as serum glutamic pyruvic transaminase (sGPT), serum glutamic oxaloacetic transaminase (sGOT), serum alkaline phosphatase (sALP), serum bilirubin (SB), thiobarbituric acid reactive substances (TBARS), $\gamma$-glutamyl transferase (GGT), lactate dehydrogenase (LDH), total protein (TP), albumin. The physical parameters including liver weight, body weight and histopathological changes in the liver were studied with Ursodeoxycholic acid as standard hepatoprotective agents. Results: The phytochemical investigation of the extracts showed the presence of Alkaloids, volatile oil, saponin glycosides, condensed tanins, terpenoids, steroids and flavonoids. Methanol and aqueous extract before the paracetamol administration caused a significant reduction in the values
\end{abstract}

of sGOT, sGPT, sALP, TBARS, GGT, LDHTP, Albumin and sB $(P<0.01)$ almost comparable to the Ursodeoxycholic acid. The hepatoprotective activity was confirmed by histopathological examination of the liver tissue of control and treated animals. Conclusions: The result concludes that Prunus armeniaca L. possesses the hepatoprotective effect against paracetamol induced liver toxicity in rats.

Key words: Apricot, Hepatotoxicity, Paracetamol, Liver toxicity.

Correspondence:

Ms.Varsha Raj, Department of Pharmacy, Pharmacology Research Lab, IFTM University, Moradabad, 244102, INDIA.

Email: varsharaj01@gmail.com

DOI : 10.5530/pj.2016.2.9

\section{INTRODUCTION}

Prunus armeniaca L. (Apricot or khubbani) belonging to family Rosaceae is an important edible, medicinal plant which is reported to contain polysaccharides, polyphenol, fatty acid, sterol derivatives carotenoids, carcinogenic glycosides, volatile component. The reported activities for this plant include antimicrobial, antimutagenic, cardio protective, anti-inflammatory, antinociceptive antioxidant activity. In very small amounts, the hydrogen cyanide present in apricot kernels has been traditionally prescribed in Chinese medicine for treating asthma, cough, and constipation.

In present time, many newly developed drugs (e.g., Rimonabant, Propylthiouracil, or Corticosteroids) have been used for treatment of liver diseases; however, these drugs possess harmful side effects such as insomnia, vomiting, constipation, and depression. For that reason, further research on plants and herbs that could potentially substitute the chemical-based drugs are very crucial as many medicinal plants have been found to possess hepatoprotective properties. ${ }^{2}$

The liver is vital organ of paramount significance involved in the maintenance of metabolic functions and detoxification of the exogenous and endogenous challenges like xenobiotic drugs, viral infections and chronic alcoholism. It is involved in almost all the biochemical pathways related to growth, to fight against disease, nutrient supply, energy provision and reproduction. ${ }^{3}$ Diverse homeostatic mechanisms are affected if liver functions are impaired with serious consequences. ${ }^{4}$ Almost all types of liver injuries may lead to hepatic failure and ultimately death. Thus liver diseases are one of the most fatal diseases in the world today. ${ }^{5}$ It afflicts over $10 \%$ of the world population. This constitutes hepatitis, cirrhosis, fibrosis, hepatic steatosis (fatty liver) alcoholic liver disease and drug induced liver disease. Morbidity and mortality resulting from liver diseases is a major public health problem worldwide, especially in developing countries. ${ }^{6}$ Most of the hepatotoxic chemicals damage liver cells primarily by producing reactive species, which form covalent bond with the lipids of the tissue. However, inbuilt protective mechanisms combat the hazardous reactions associated with the free radicals. Due to excessive exposure to hazardous chemicals, sometimes the free radicals generated are so high that they overpower the natural defensive system leading to hepatic damage and cause jaundice, cirrhosis and fatty liver. ${ }^{7}$

Paracetamol (acetaminophen) is a widely used antipyretic and analgesic, which produces acute liver damage if overdoses are consumed. Paracetamol is mainly metabolized in the liver to excretable glucuronide and sulphate conjugates. ${ }^{8,9}$ At toxic doses of Paracetamol, the normal metabolic pathways become saturated, causing both more $\mathrm{N}$-acetylp-benzoquinone imine (NAPQI) to be formed and hepatic glutathione to be rapidly depleted. Paracetamol toxicity is caused by the reaction metabolite, $\mathrm{N}$-acetyl-p-benzoquinoneimine (NAPQI), which is partly metabolized by cytochrome P- $450 .{ }^{10}$ This species causes severe oxidative damage and glutathione depletion leading to liver necrosis. Introduction of cytochrome or depletion of hepatic glutathione is a prerequisite for paracetamol-induced hepatotoxicity. ${ }^{11,12}$ The survey of literature reveals that the Prunus armniaca L. is one of the most important Indian medicinal plants. Traditionally, it is used as an antimicrobial, antimutagenic, Antituberclousis, cardio protective, anti-inflammatory, antinociceptive and antioxidant. Presence of hydrogen cyanide in very small amount is also reported in earlier study in apricot kernels, which has traditionally prescribed in Chinese medicine for treating asthma, cough, and constipation. Furthermore, it is used in cosmetic industry as a astringents, for cooling effect on irritated skin, Its is also claimed to increase the fertility. ${ }^{13}$ The present study was aimed to evaluate the hepatoprotective potential of Prunus armeniaca L. (Leaves) against paracetamol-induced hepatotoxicity.

\section{MATERIAL AND METHODS}

\section{Drugs and chemicals}

Paracetamol was obtained from the Ranbaxy laboratory limited as a gift sample and Ursodeoxycholic acid was purchased from Embee diagnoses, Serum glutamic oxaloacetic transaminase (SGOT), Serum glutamic pyruvic transaminase (SGPT) (ERBA Diagnostics, Mannheim GmbH 
Transasia bio-medical Ltd), Total bilirubin (Span diagnostics Ltd)-Surat. Other chemicals used in this experiment were also of analytical grade.

\section{Plant material}

The fresh leaves of Prunus armeniaca L. were collected in the month of July from Dist. Sirmour. Himachal Pradesh (India) and authenticated by NISCAIR New Delhi, India and the herbarium file of this plant (Voucher no -NISCAIR/RHMD/Consult/2011-12/1918/218) was submitted to the herbarium section of NISCAIR for future reference.

\section{Preparation of extracts}

The fresh leaves were cleaned, shade dried and then powdered. The dried coarse powder of the leaves ( $1000 \mathrm{~g}$ ) was extracted by using the soxhlet apparatus to perform methanolic and aqueous extractions. Thereafter extracts were wiped in a vacuum according to the distillation method (Freeze Dryer). The extracts were preserved by placing to sample bottles wrapped with aluminum foil and kept at $4^{\circ} \mathrm{C}$. The extracts were subjected to preliminary phytochemical investigation. Extracts were dissolved in water for injection (I.P), Paracetamol ( $3 \mathrm{~g} / \mathrm{kg}$ ) suspension prepared by using 40\% Sucrose solution and subjected for hepatoprotective activity against paracetamol-induced liver damage.

\section{Phytochemical Analysis}

The methanolic and aqueous extracts were subjected to the phytochemical analysis using conventional protocol like alkaloids, flavonoids, carbohydrates, glycosides, saponins, tannins, proteins, aminoacids, fixed oils, mucilage etc. ${ }^{14}$

\section{Experimental animals}

A study was carried out by using Albino-Wistar rats either by sex, weighing 150 to $200 \mathrm{~g}$. They were obtained from the animal house, IFTM University, Moradabad, India. The animals were grouped and housed in polyacrylic cages with not more than six animals per cage and maintained under standard laboratory condition (temperature $25 \pm 2)^{\circ} \mathrm{C}$, with dark and light cycle $(12 / 12 \mathrm{~h})$. The animals were fed with standard pellet diet supplied by Hindustan Lever Ltd and fresh water ad libitum. All the animals were acclimatized to laboratory condition for a week before commencement of experiment. All procedures described were reviewed and approved by the University Animal Ethics Committee.

\section{Acute oral toxicity}

The acute oral toxicity study was carried out as per the guidelines set by the OECD, revised draft guidelines 423, received from CPCSEA, Ministry of Social Justice and empowerment, Govt. of India. ${ }^{15}$

\section{Evaluation of hepatoprotective activity}

The method used for evaluation of hepatoprotectivity was performed as per method described by Maity et al, ${ }^{16}$ Mayuren $\mathrm{C}$ et al, ${ }^{17}$ Hegde $\mathrm{K}$ et $a l^{18}$ with some minor modifications. In the paracetamol-induced liver toxicity model, paracetamol ( $3 \mathrm{~g} / \mathrm{kg}$, p.o.) was administered on the 8th day to all animals except group 1. Paracetamol was suspended in ( $40 \% \mathrm{w} / \mathrm{v})$ sucrose solution. Ursodeoxycholic acid $(250 \mathrm{mg} / \mathrm{kg}$, p.o.) was used as a standard. The animals were segregated into five groups of six each. Group 1, which served as normal control and received $5 \mathrm{ml} / \mathrm{kg}$ b.w; p.o saline water daily for 7 days. Group 2 treated as group 1 for 7 days and served as negative control. Group 3 received Ursodeoxycholic acid (250 mg/kg, p.o.) for 7 days. Group 4 received a methanol extract (200 mg/kg, p.o.) for 7 days. Group 5 received an aqueous extract (200 mg/kg, p.o.) for 7 days. The animals were sacrificed $48 \mathrm{~h}$ after paracetamol administration under light anesthetic ether. Blood from each rat was withdrawn by retro orbital plexus under ether anesthesia for biochemical investigation of parameters such as i.e. sGOT, sGPT, sALP, LDH, TB, TBARS, GGT, Total Protein, Albumin. Serum was sepa- rated by the centrifugation of blood at $2500 \mathrm{rpm}$ for $15 \mathrm{~min}$ at $40^{\circ} \mathrm{C}$ and supernatants were collected which were further used for the study of biochemical parameters. The liver of all the experimental animals was removed and processed immediately for histological investigation. ${ }^{17}$

\section{Histopathological studies}

The livers were excised quickly and fixed in $10 \%$ formalin and paraffin embedded. Sections of about 4- $6 \mu \mathrm{m}$ were stained with haemotoxylin and eosin ( $\mathrm{H} \& \mathrm{E})$ for the liver histopathological architecture. In brief, 4-6 $\mu \mathrm{m}$ thick sections of paraffin embedded rat liver were dewaxed with distilled water for $2 \mathrm{~min}$. Then the sections were stained with haemotoxylin for $5 \mathrm{~min}$ at room temperature. After $15 \mathrm{~min}$, the sections were counterstained with eosin for $2 \mathrm{~min}$, dehydrated with alcohol, washed with xylene and blocked by eosin. Hemotoxylin and eosin stained studies were observed under microscope. The staining procedure for histology was done by hematoxylin, a basic dye stains nuclei blue and eosin, an acidic die, stains pink color to the cytoplasm.

\section{Statistical analysis}

All the results obtained were expressed as mean \pm SD (Standard deviation) and analyzed by one-way ANOVA, followed by Dunett ' $t$ ' test between groups using Graphpad Instat version 3.06 (GraphPad Software, Inc. CA, USA). The results of all the extracts including the standard drug are compared with the result produced by normal group, and it is considered as significant as $\mathrm{p}<0.05$.

Data are presented as means + SEM $(n=6)$, one way Annova followed by Dunett's t test.

${ }^{\star} \mathrm{p}<0.05,{ }^{* *} \mathrm{p}<0.01$ vs.paracetamol treated group.

\section{RESULTS}

Extracts subjected for phytochemical study showed the presence of alkaloids, volatile oil, saponin glycosides, condensed tanins, terpenoids, steroids and flavonoids in both extracts.

Acute toxicity Methanolic and aqueous extracts did not show any sign and symptoms of toxicity and mortality up to $2000 \mathrm{mg} / \mathrm{kg}$ dose.

\section{Hepatoprotective activity}

The hepatoprotective activity of methanolic and aqueous extracts of Prunus armeniaca L. on Paracetamol treated rats are showed in Table 1. Increase in the serum hepatic enzyme levels, such as sGOT, sGPT, sALP, LDH, TB and GGT by the Administration of paracetamol $3 \mathrm{~g} / \mathrm{kg}$, p.o) was compared to normal groups showed the marked increased value. While the other parameters such as albumin and total protein showed the marked reduction in the levels of liver enzymes as compared to the normal groups indicating liver damage. Body weight and liver weight also showed the significant results that indicate chronic liver damage.

\section{Histopathological results}

The hepatic architecture was present in normal group with features of polygonal nucleus with nucleolus, abundant cytoplasm and bilobed nucleus and showed no visible changes, disarrangement in hepatic cells (Figure 1), these normal structures were absent in toxic control group in which there were macrovesicular steotosis, centrilobular necrosis and degeneration were found (Figure 2). The histopathological profile of the rat treated with methanol extract showed no visible changes confirming the safety of the extract at a selected dose (Figure 4 ). The liver section of treated group with aqueous extract and intoxicated with paracetamol showed moderate hepatoprotective activity (Figure 5). While the standard group treated with Ursodeoxycholic acid and intoxicated with paracetamol showed less disarrangement and degeneration of hepatocytes, indicating marked regeneration activity (Figure 3 ). 


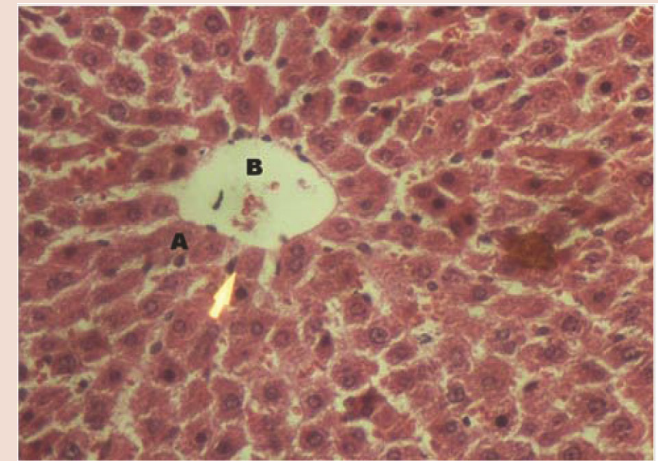

1

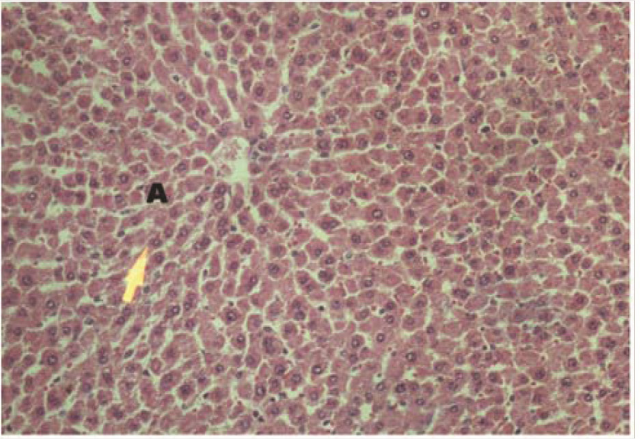

3

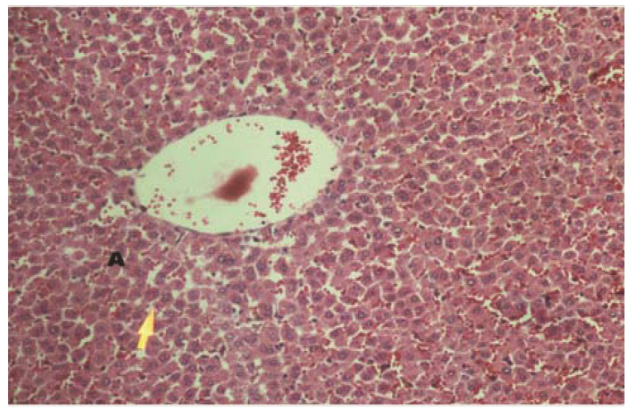

5

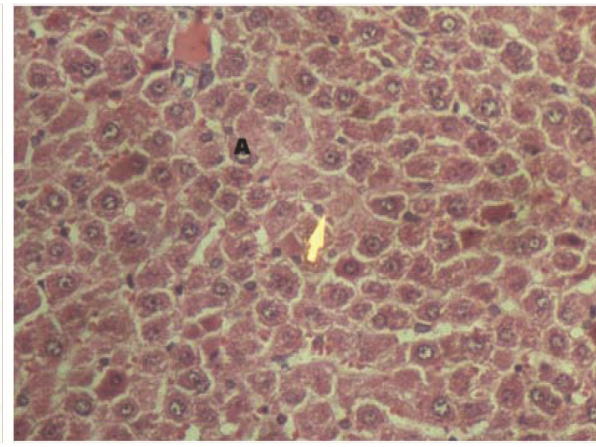

4

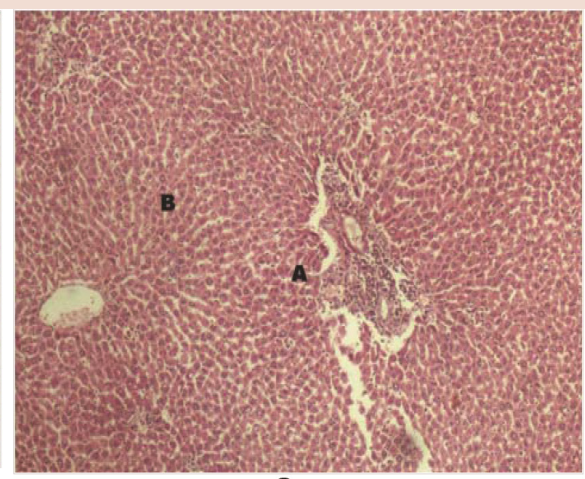

2

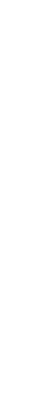

Figure 1-5: Histological monogaraph of extract and standard 1.Normal; 2.Paracetamol (3g/kg); 3.Paracetamol + Ursodeoxycholic acid $(250 \mathrm{mg} / \mathrm{kg}) ; 4$. Paracetamol + Methanolic extract $(200 \mathrm{mg} / \mathrm{kg})$; 5. Paracetamol + Aqueous extract ( $200 \mathrm{mg} / \mathrm{kg}$ ) 


\section{DISCUSSION}

Hepatotoxicity is the potential complication of paracetamol, which is widely, used in general medicine and an assessment of its relative toxicity is important. The primary toxicity of paracetamol is the result of drug metabolism in liver. ${ }^{18}$ At therapeutic doses, paracetamol is metabolized via glucuronidation and sulfation reactions result in the water-soluble metabolites that are excreted via the kidney. The result of the metabolic conversion of paracetamol by the microsomal P-450 enzyme system is that, a highly reactive intermediate, namely, N-Acetyl-P Benzoquinone Imine (NAPQI) is produced. Glutathione $(\mathrm{GSH})^{19}$ then reduces this metabolite.

However, after Paracetamol overdose, capacity for glucuronidation and sulfation is exceed with the formation of excess NAPQI via cytochrome P450 2E1.

This intern lead to depletion in glutathione, excess of NAPQI binds to hepatic cell protein and DNA resulting in mitochondrial dysfunction and the development of acute hepatic necrosis.$^{20}$

More ever NAPQI can also make covalent bound with cellular proteins, can change the structure and functions of these proteins. This cellular damage causes a decrease in calcium ATPase activity and an increase in cytosolic calcium levels. As a result, cell permeability changes and lead to the loss of cellular integrity. ${ }^{21}$ In addition, it has been shown that NAPQI can inhibit NADH and succinate dehydrogenase function. It was considered that destruction of homeostasis was also responsible for high dose paracetamol induced hepatotoxicity. Because of destruction of homeostasis, intracellular $\mathrm{Ca}^{2+}$ accumulates and an increase is shown in catabolic enzymes, which causes cell death. Nitric oxide, reactive oxygens, lipid peroxidation and apoptosis are the major situations, which play roles in hepatotoxicity. In addition, peroxinitrite derivatives and protein nitrates also have roles in this condition. ${ }^{22}$ So in the present study, paracetamol was employed as toxic agent and the protective effect of Prunus armeniaca L. leaves against the paracetamol induced hepatotoxicity was studied. Histopathological studies and biochemical enzyme markers like sGOT, sGPT, SALP, TB, LDH, GGT, Total protein and albumin levels estimated the extent of toxicity.

Reinforcing the above stated mechanisms, The histopathological study also supported the biochemical evidence for the hepatoprotection shown by Prunus armeniaca L. in the same group. The extent of hepatic damage is assessed by histological evaluation and the level of various biochemical parameters significant increase in the toxic control groups in the present study. The hepatic damage associated with Paracetamol is due to release of toxic metabolite $\mathrm{N}$-acetyl-P-benzoquinone imine (NAPQ1) and its free radical generation. The protective effect exhibited by both methanolic and aqueous extracts at a dose level of $200 \mathrm{mg} / \mathrm{kg}$ was comparable with the standard drug Ursodeoxycholic acid. The methanol extract showed more significant results as compared to aqueous extract Prunus armeniaca L. leaves.

After the phytochemical study, it is reported that, the plant extracts contain flavonoids, terpenoids, tannins and steroids. The presence of flavanoids in our extract may be responsible for its hepatoprotective activity. ${ }^{23-7}$ In conclusion, the results of this study demonstrate that Prunus armeniaca L. extract was effective for the prevention of Paracetamol-induced hepatic damage in rats. Our results show that the hepatoprotective effects of Prunus armeniaca L. extract may be due to marked changes in various parameters of liver enzyme, some physical parameters which proved that it's a potent hepatoprotective in nature against the Paracetamol induced liver toxicity.

The present study thus justifies the use of Prunus armeniaca L. in the treatment of liver diseases and points out that Prunus armeniaca L. warrants further detailed investigation as a promising hepatoprotective agent does. However, the exact mechanism(s) and the active compound(s) involved in these effects need to be clarified in future studies.

\section{CONFLICT OF INTEREST}

We declare that we have no conflict of interest.

\section{ABBREVIATION USED}

SGOT: Serum oxaloacetate transminase, SGPT: Serum pyruvate transminase, TP: Total protein, ALP: Alkaline phosphatase, Al: Albumin, LDH: Lactate dehydrogenase, TB: Total bilirubin, GGT: $\gamma$-glutamyl transferase, L/W: Liver weight, B/W: Body Weight, U/L: International units/litre.

\section{REFRENCES}

1. Erdogan-Orhan I, Kartal M, Insight in to research on phytochemistry and biological activites of Prunus armeniaca L.(apricot), Food Research International. 2011;44(5):1238-43

2. Mahmood N D, Mamat S S, Kamisan F H, Yahya F, Kamarolzaman M F F, Nasir N, Mohtarrudin N, Tohid S F Md, Zakaria Z A. Amelioration of Paracetamol-Induced Hepatotoxicity in Rat by the Administration of Methanol Extract of Muntingia calabura L. Leaves. Bio Med Research International. 2014; 1-10.

3. Drug development for liver disorders and hyperlipidemia. 2012

4. Ganguly P, Gupta A K, Majumdar U K, Ghosal S. Hepatoprotective and antioxidant effect of naturally occurring withasteroid metal ion conjugates of Withania sominefera in Paracetamol induced Hepatotoxicity in rats. Pharmcologyonline. 2009;1:1044-56

5. Dinesh K, Sivakumar V, Selvapriya B, Deepika E, Mohamed Sadiq A. Evaluation of hepatoprotective polyherbal formulations contains some Indian medicinal plants. Journal of Pharmacognosy and Phytochemistry. 2014; 3(4):1-5.

6. Syed SH, Namdeo A G. Current status of natural products for the treatment of liver disease-A review. International Journal of Phytopharmacy. 2014;4(2):37-43

7. Meganathan M, Madhana MG,Sasikala P, Mohan J, Gowdhaman N, Balamurugan K. Evaluation of antioxidant effect of omega 3- fatty acid against paracetamol induced liver injury in albino rat. Global J Pharmacol. $2011 ; 5(1): 50-53$

8. Jyoti TM, Prabhu K, Lakshminarasu S, Ramachandra SS, Hepatoprotective and antioxidant activity of Euphorbia tirucalli. Iranian J of Pharmacology. 2008;7(1):25-30

9. Jollow DJ, Thorgerisson SS, Potter WZ, Hashimoto M, Mitchell JR, Acetaminophen induced Hepatic necrosis VI. Metabolic disposition of toxic and non-toxic doses of acetaminophen. Pharmacology. 1974;12(4-5):251-71.

10. Wong LT, Whitehouse LW, Solemonraj G, Paul CJ.Pathways of Acetaminophen conjugate in the mouse. Toxicity Lett 1. 1981;9:145-51.

11. Dahlin CD, Miwa G, Lu A, Nelson S. N-acetyl-p-benzoquinoeimine: A cytochrome P-450-mediated oxidation product of acetaminophen. Proc Natl ACAD Sci 1984; $81(5): 1327-31$.

12. Moron MS, Depierre JW, Mannervik B. Levels of glutathione, glutathione reductase and glutathione-S-transferase activities in rat lung and liver. Biochem Biophys Acta. 1979; 582(1):67-78.

13. Sehgal J, Siddheswaran $P$, Senthil Kumar K L, Karthiyayini T. Anti-tubercular Activity of fruits of Prunus armeniaca (L.). International Journal of Pharma and Bio Sciences. 2010:1(2):1-4.

14. Handa SS. Future trends of plants as drugs. Pharma Times. 1991;23(4):13-23.

15. Organisation for Economic Co-operation and Development Guidelines on acute oral toxicity. Revised Document. October 2005.

16. Maity TK, Maiti BC, Sengupta P, Rajalingam D, Ghosh T, Nayak S.S, Yeligar VC, Dash DK. Evaluation of hepatoprotective and antioxidant activity of Lchnocarpus frutescens (Linn.) R.Br.on paracetamol induced hepatotoxicity in rats. Tropical J of Pharmaceutical Research. 2007;6(3):755-65.

17. Mayuren C, Reddy VV, Priya SV, Devi VA. Protective effect of Livactine against $\mathrm{CCl} 4$ and paracetamol induced hepatotoxicty in adult wistar rats. North American $\mathrm{J}$ of Medical Science. 2010; 2(10): 491-95.

18. Hegde K, Joshi AB. Hepatoprotective effect of Carissa carandas Linn root extract against $\mathrm{CCl} 4$ and Paracetamol induced hepatic oxidative stress. Indian J of Experimental Biology. 2009; 47(8): 660-67.

19. Fedekar F, Madkour, M. M. Abdel-Daim. Hepatoprotective and Antioxidant Activity of Dunaliella salina in Paracetamol-induced Acute Toxicity in Rats. Indian J Pharm Sci. 2013; 75(6): 642-48.

20. Elmhdwi MF, Muftah SM, Tumi SGE, Elslimani. Hepatoprotective effect of Ecballium elaterium fruit juice against paracetamol induced hepatotoxicity in male albino rats. International current Pharmaceutical J. 2014; 3(5):270-74. 
21. Bigoniya P, Singh CS, Shukla A. A comprehensive review of different liver toxicant used in experimental pharmacology. Inte $\mathrm{J}$ of Pharmaceutical Science and Drug research. 2009; 1(3):124-35.

22. Jaeschka A, Abbas B, Zabel M, Hopmans EC, Schouten S, Damste JSS. Molecular evidence for anaerobic ammonium-oxidizing (anammox) bacteria in continental shelf and slope sediments off northwest Africa. American Society of Limnology and ocenology. 2010;55(1):365-76.

23. Mustfa M, Emre K, Abdulmecit A, Lihami K, Yasin B, Elif C, Deniz U, Zekai H, Ali K. Protective effect of Panax giniseng against N-acetyl-P-aminophenol-induced hepatotoxicity in rats. African J of Pharmacy and Pharmacology. 2012; 6:2634-42.

24. Soni RA, Irchhaiya R, Dixit $V$, Bhat ZA, Wani HA, Najar AH. Hepatoprotective activity of Kirgangella reticulata poir (Baile) root against paracetamol induced hepatotoxicity in wistar rats. Int $\mathrm{J}$ of Pharmacy and Pharmaceutical Science. 2014;6:273-78.

25. Pal A, Banerjee B, Banerjee T, Masih M, Pal K. Hepatoprotective activity of Chenopodium album Linn plant against Paracetamol-induced hepatic injury in rats. Int Journal of Pharmacy and Pharmaceutical Science 2011; 3(3):55-7.

26. Yadav NP,Pal A, Shanker K, Bawankule DU, Gupta A K, Darokar MP Khanuja SPS. Synergestic effect of Silymarin and standardized extract of Phyllanthus amarus against $\mathrm{CCl}$-induced hepatotoxicity in Rattus norvegicus. Int J of Phytotherapy and Phytopharmacology. 2008; 15(12):1053-61.

27. More AM, Parab PB, Apte KG. Evaluation of activity of whole stem extracts of Oroxylum indicum on Paracetamol induced hepatotoxicity. Int J of Pharma and Biosciences. 2013;4:255-65.

\section{PICTORIAL ABSTRACT}

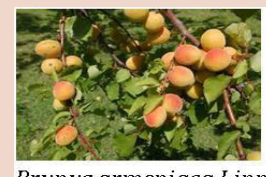

Prunus armeniaca Linn

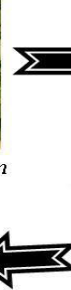

Reduced Liver

damage and

increased

hepatoprotection

\section{SUMMARY}

- The phytochemical study of the plant is reported that, the extracts contain flavonoids, terpenoids, tannins and steroids. The presence of flavanoids in our extract may be responsible for its hepatoprotective activity.

- The present study, paracetamol was employed as toxic agent and the protective effect of Prunus armeniaca L. leaves against the paracetamol induced hepatotoxicity was studied.

- Histopathological studies and biochemical enzyme markers like sGOT, sGPT, SALP, TB, LDH, GGT, Total protein and albumin levels estimated the extent of toxicity. The hepatic damage associated with Paracetamol is due to release of toxic metabolite $\mathrm{N}$-acetyl-P-benzoquinone imine (NAPQ1) and its free radical generation.

- The protective effect exhibited by both methanolic and aqueous extracts at a dose level of $200 \mathrm{mg} / \mathrm{kg}$ was comparable with the standard drug Ursodeoxycholic acid. The methanol extract showed more significant results as compared to aqueous extract Prunus armeniaca L. leaves.

\section{ABOUT AUTHORS}

Ms. Varsha Raj: Obtained her M. Pharm degree in Pharmacology in 2012 from Maharishi Markendeshwar University, Ambala Haryana. She is currently working on the post of Assistant Professor in Pharmacy Academy, IFTM University Moradabad. She works in the area of hepatoprotective activity of herbal plant in pharmacology.

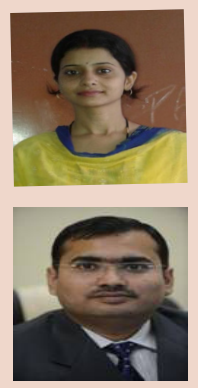

Dr. Amrita Mishra: Phd from Birla institute of technology, Ranchi is working as a Assistant Professor at School of Pharmaceutical Sciences, IFTM University, Mordabad. She works in the area of Herbal drug standardization, Phytochemistry and Pharmcology. She have great knowledge of writing projects and research papers.

Dr. Arun K Mishra: Recipient of fellowship from All India Council of Technical Education (AICTE) Govt. of India for M.Pharm. He is also recipient of Shiri Devi Memorial award from IFTM administration for best performance in P.G Course. Currently, he is working as Assistant Professor in School of Pharmaceutical Sciences. IFTM University, Moradabad, He has 39 research papers in national and international journals and 19 poster presentations in various National and Internatonal conferences. Presently he is supervising $6 \mathrm{Ph}$. D research scholars.

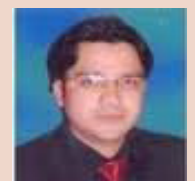

Dr. Najam Ali Khan: Phd from UPTU Lucknow. Working as a Associate Professor at School of Pharmaceutical Sciences, IFTM University, Mordabad. He works in the area of cardioprotecive activity of herbal drugs in Pharmcology. He have great knowledge of writing projects and research papers. He has 19 research papers in national and international journals and 10 poster presentations in various National and Internatonal conferences. Presently he is supervising $8 \mathrm{Ph}$. D research scholars. 